\title{
Spectrum of histopathological changes in fibroadenoma of breast
}

\author{
Jehan Nizam Ansari'1, Archana Chirag Buch ${ }^{2, *}$, Aditi Pandey ${ }^{3}$, Ruby Rao $^{4}$, Ayesha Siddique ${ }^{5}$ \\ 1,3,4,5Resident, ${ }^{2}$ Professor, Dept. of Pathology, Dr. D.Y. Patil Medical College Hospital and Research Centre, Dr D.Y. Patil \\ Vidyapeeth, Pimpri, Pune, Maharashtra, India
}

*Corresponding Author:

Email: drarchanabuch@yahoo.co.in

Received: $18^{\text {th }}$ November, 2017

Accepted: $2^{\text {nd }}$ April, 2018

\begin{abstract}
Introduction: Fibroadenoma commonly known, as 'Breast Mouse' is freely mobile, benign tumour of the breast. It is composed of epithelial and stromal components. These components may show varied histomorphological changes. The aim of the present study is to explore the histopathological features of the epithelium and stroma within and around breast fibroadenoma.

Materials and Methods: This is a retrospective and prospective unicentre study on total 317 cases of fibroadenoma over a period of two years. Formalin fixed specimens were grossed and stained with routine H\&E. Further detailed histopathological examination was done.

Results: Simple features were noted in 94\% whereas 6\% showed complex features. On detailed HPE, proliferative epithelial changes like mild ductal hyperplasia was seen in $73.5 \%$ of cases followed by moderate ductal hyperplasia seen in $12.6 \%$. Among the fibrocystic epithelial changes apocrine metaplasia, cystic changes and calcification were observed in $35.91 \%, 18.3 \%$ and $5 \%$ cases respectively. Only 71 cases had adjacent parenchyma revealing insignificant changes. Increasing age had statistically significant relationship with complex features. This is statistically significant with $\mathrm{p}$ value $<0.001$. Duration of the illness was insignificant with respect to simple and complex features.

Conclusion: Histopathological Examination of fibroadenomas should be done thoroughly to see atypical, complex changes or any malignant feature. Further follow up should be recommended in cases with proliferative changes for early detection of malignancy.
\end{abstract}

Keywords: Fibroadenoma, Histopathological changes, Malignant transformation.

\section{Introduction}

Fibroadenoma is a freely mobile, firm tumour of breast occurring in woman of reproductive age group. They are usually benign biphasic tumors made up of both epithelial and stromal components. ${ }^{1,2}$ Depending on the proportion and the relationship between these two components, there are main three histological types: Intracanalicular, pericanalicular and mixed. Intracanalicular pattern shows stromal proliferation compressing the ducts into slit like spaces. ${ }^{3}$ In pericanalicular fibroadenoma fibrous stroma proliferates around the ductal spaces so that they remain round or oval, on cross section. When both the features were present, they were quoted as mixed. ${ }^{3}$

Microscopically fibroadenoma can be divided into two categories: Simple fibroadenomas, they are often less cellular, do not increase the risk of breast cancer. Complex fibroadenomas contains other components such as epithelial calcifications, apocrine metaplasia, sclerosing adenosis, and cysts larger than $3 \mathrm{~mm}$ in diameter. Complex fibroadenomas can slightly increase the risk of breast cancer. ${ }^{3}$

Malignant transformations in the epithelial components of fibroadenomas are generally considered rare. ${ }^{4}$ However in very few cases malignant transformation has been reported on histopathological examination. ${ }^{5}$ Proliferative changes in the fibroadenoma were related to a further increase of risk by $3.88 .{ }^{6}$ The aim of the present study is to explore the histopathological features of the epithelium and stroma within and around breast fibroadenomas.

\section{Materials and Methods}

This is a retrospective and prospective unicentre study on spectrum of histopathological changes in fibroadenoma of breast carried out at Dr. DY Patil Medical College Hospital and research Centre. Study was conducted from July 2015 to September 2017. Total number of cases collected was 317 . Formalin fixed excisional breast biopsies of patients, with histopathological diagnosis of fibroadenoma was included in the study. Autolysed specimens, breast lumps in pregnancy, male patient and patient with known malignancy were excluded from the study. Data for the study was obtained from departmental records, tissue block slides, and clinical records. Clinically suspected fibroadenoma received as incisional or excisional breast biopsies from the department of surgery were fixed in $10 \%$ formalin, for histopathological examination (HPE). Formalin fixed specimens were grossed and stained with routine $H \& E$ staining and special stains wherever indicated. Ductal hyperplasia was diagnosed using the criteria of Page et al and Holland et al. ${ }^{7}$ A care was taken not to mistake an artificial hyperplasia like pattern caused by detachment of the epithelium with subsequent curling up, leading to a widened duct filled with epithelial strands. ${ }^{8}$ The distinction between normal hyalinized or 
collagenized stroma and smooth muscle changes was done with the help of Masson's Trichrome staining. Rosen's criteria were used to distinguish phyllodes tumor from fibroadenoma. ${ }^{9}$

Detailed HPE was done for epithelial and stromal changes as shown in Table 1. Adjacent parenchyma was seen only in 71 cases. Histopathological changes in adjacent parenchyma were observed as shown in Table 2. Various histomorphological changes seen are as shown in Fig. $1 \& 2$. Appropriate statistical tests like chi square test for categorical variables in proportions and for continuous variables $\mathrm{T}$ test and ANOVA were applied. $\mathrm{P}$ value less than 0.001 was taken as significant.
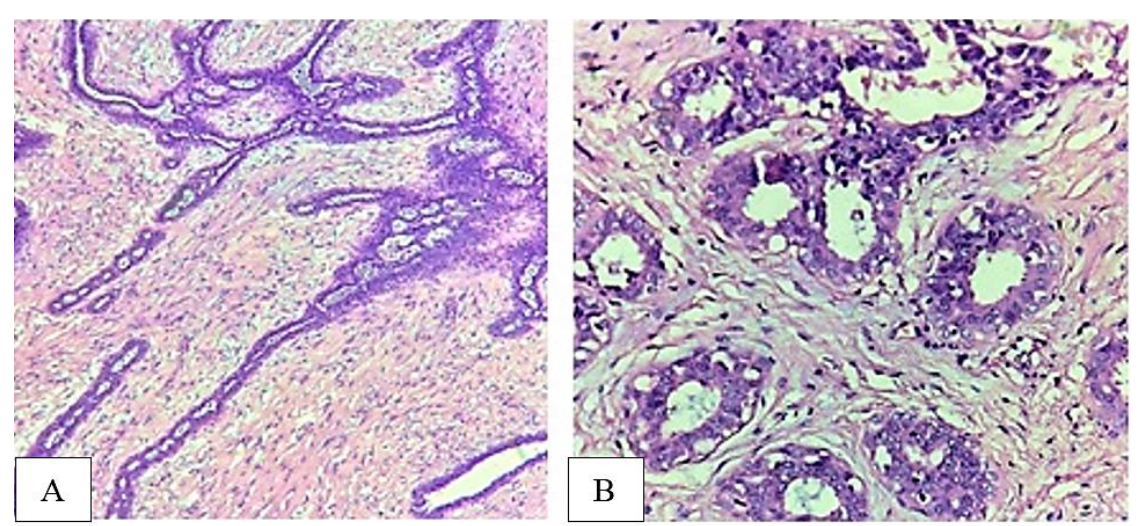

Fig. 1A: Intracanalicular pattern of fibroadenoma (H\&E, x40); B): Moderate epitheliosis with myxoid stroma (H\&E, x40)
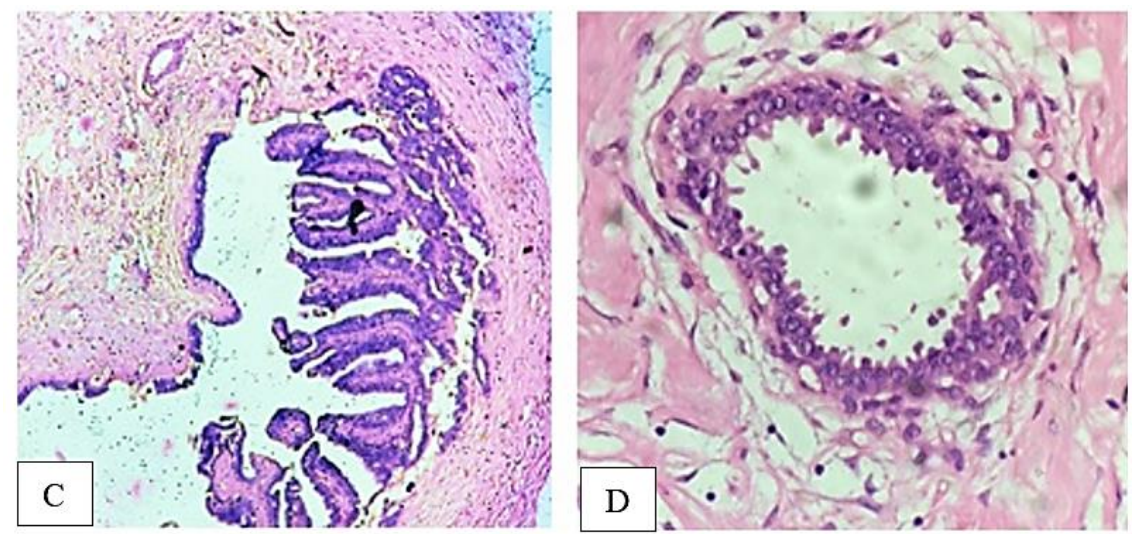

Fig. 1C: Papillary projection in Complex fibroadenoma (H\&E, x40); D): Apocrine metaplasia seen in fibroadenoma (H\&E, x100)
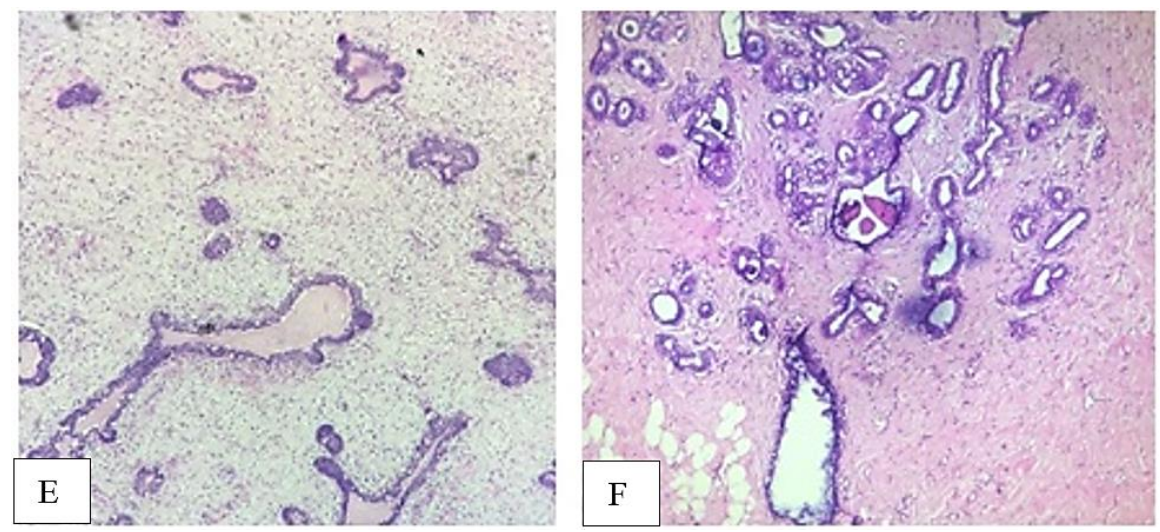

Fig. 1E: Fibrocystic changes seen in fibroadenoma (H\&E, x40); F): Calcification seen in complex fibroadenoma 

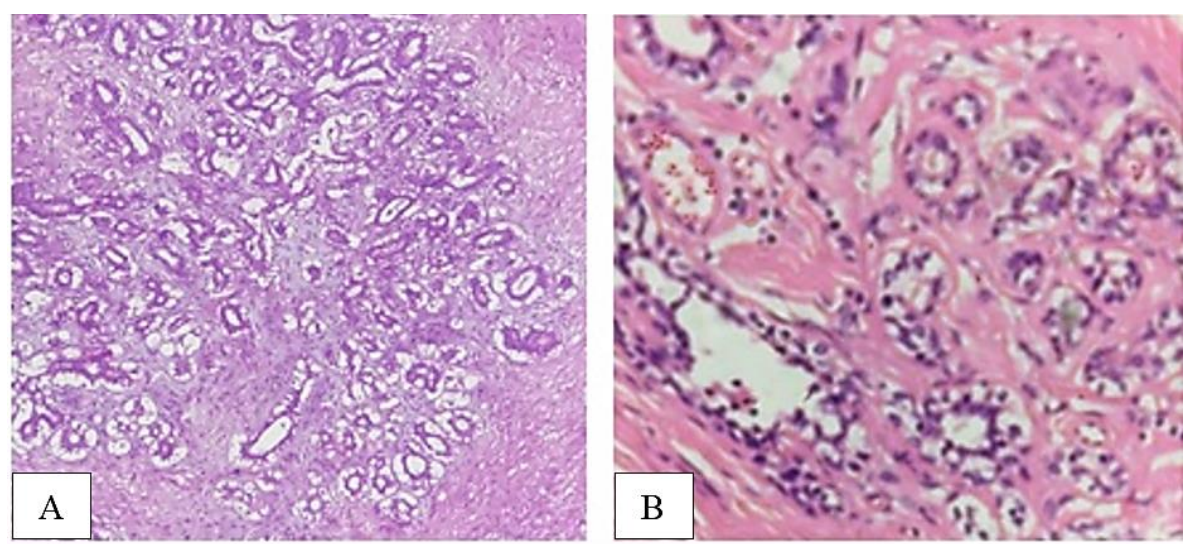

Fig. 2A: Sclerosing adenosis seen in Fibroadenoma (H\&E, x40); B): Tubular adenosis seen in fibroadenoma $(\mathbf{H} \& \mathbf{E}, \mathbf{x 4 0 )}$
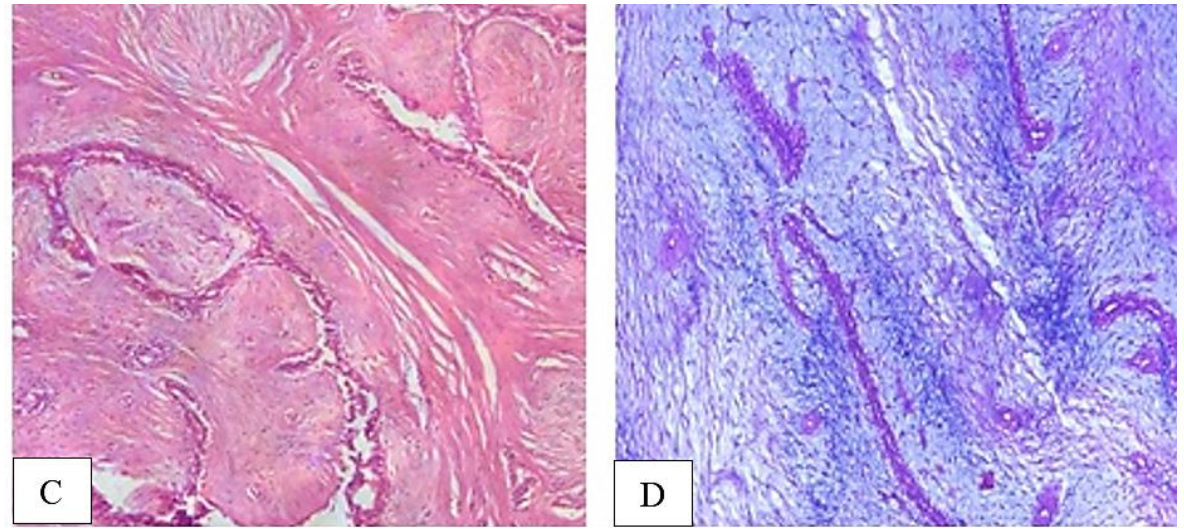

Fig. 2C: Extensive hyalinization seen in fibroadenoma (H\&E, x40); D): Myxoid changes seen in fibroadenoma adenoma (H\&E, x40)
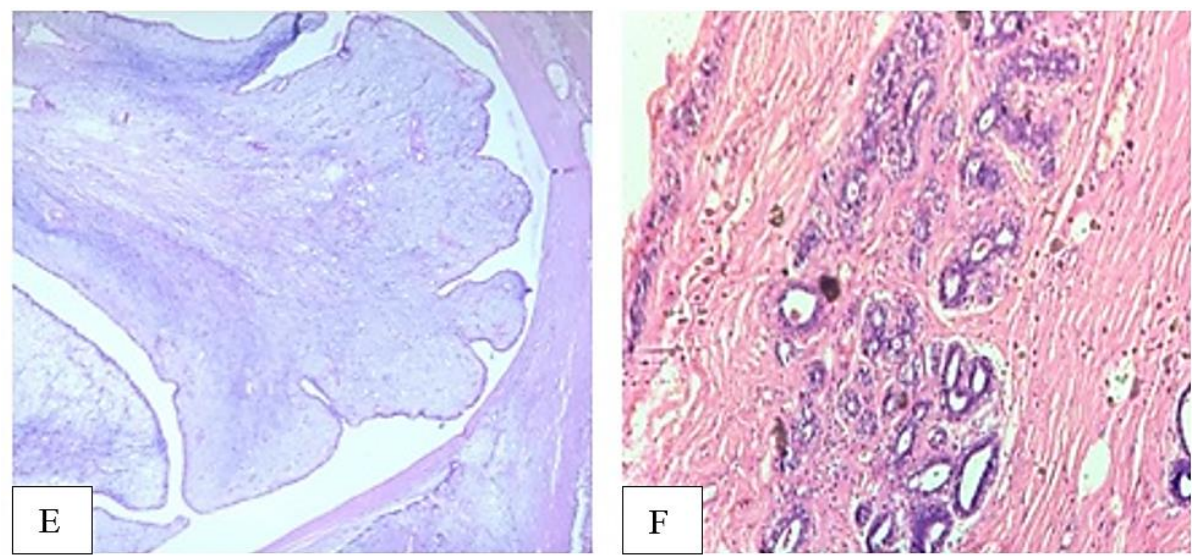

Fig. 2E: Phyllodes tumor in adjacent parenchyma of fibroadenoma (H\&E, x40); F: Adjacent parenchyma showing tubular adenosis in fibroadenoma $(\mathrm{H \& E}, \mathbf{x 4 0})$

\section{Results}

The mean age of study population was 27.9 years. Most of the cases were noted in second and third decades of life. 94\% $(\mathrm{n}=298)$ cases reflected simple fibroadenomas whereas only $6 \%(n=19)$ cases were of complex nature. Complexity was observed $2.40 \%$, $12.63 \%$ and $20 \%$ in second, third and fourth decade respectively. Increasing age had statistically significant
( $\mathrm{p}$ value $<0.001)$ relationship with complex features. We encountered $83.9 \% \quad(n=266)$ cases of unilateral fibroadenomas. Right breast was more affected than the left breast. Associated masses were found in $3.47 \%$ $(n=11)$ cases. Cyclic mastalgia was found in $9.46 \%$ $(n=30)$ of cases while $13 \%(n=41)$ gave positive history of exogenous oral contraceptive pills. Recurrence was rare in our study. Significant difference was not observed in simple and complex fibroadenoma with duration of the illness. 
Table 1: Distribution of various histopathological changes in fibroadenomas

\begin{tabular}{|l|c|c|}
\hline \multicolumn{2}{|c|}{ Type Proliferative epithelial changes } \\
\hline Mild ductal hyperplasia & Frequency & Percentage (\%) \\
\hline Moderate ductal hyperplasia & 233 & 73.5 \\
\hline Florid ductal hyperplasia & 40 & 12.6 \\
\hline Atypical ductal hyperplasia & 3 & 0.9 \\
\hline Atypical lobular hyperplasia & 0 & 0 \\
\hline Lobular ca in situ & 0 & 0 \\
\hline Ductal ca in situ & 0 & 0 \\
\hline \multicolumn{2}{|c|}{ Fibrocystic epithelial changes } \\
\hline Apocrine metaplasia & 114 & 0 \\
\hline Cysts & 58 & 35.9 \\
\hline Sclerosing adenosis & 43 & 18.3 \\
\hline Calcifications & 16 & 13.6 \\
\hline Microglandular adenosis & 8 & 5 \\
\hline Papilloma & 8 & 2.5 \\
\hline Pseudo lactational changes & 4 & 2.5 \\
\hline \multicolumn{2}{|c|}{ Stromal changes } \\
\hline Pseudo angiomatous changes & 2 & 1.2 \\
\hline Myxoid changes & 204 & 0.63 \\
\hline Hyaline changes & 269 & 64.4 \\
\hline Inflammatory changes & 40 & 84.9 \\
\hline Multinucleated giant cell & 0 & 12.6 \\
\hline Smooth muscle changes & 0 & 0 \\
\hline Pericanalicular & 121 & 0 \\
\hline Intracanalicular & 100 & $38.17 \%$ \\
\hline Mixed pattern & 97 & $31.54 \%$ \\
\hline Mitotic figures & 0 & $30.59 \%$ \\
\hline Infarct & 0 & 0 \\
\hline
\end{tabular}

Table 2: Distribution of various histopathological changes in adjacent parenchyma of fibroadenoma

\begin{tabular}{|l|c|c|}
\hline \multicolumn{3}{|c|}{ Changes in adjacent parenchyma } \\
\hline \multicolumn{1}{|c|}{ Type } & Frequency & Percentage (\%) \\
\hline Foci of tubular adenoma & 9 & 12.6 \\
\hline Focal phyllodes tumour & 2 & 2.1 \\
\hline Hyaline/myxoid changes & 3 & 4.2 \\
\hline Fibrocystic changes & 19 & 26.7 \\
\hline Fat Necrosis & 11 & 15.4 \\
\hline Granulation tissue & 6 & 8.45 \\
\hline
\end{tabular}

\section{Discussion}

The study was designed to evaluate the elaborated histomorphological features of 317 cases of fibroadenoma. It is quite common to observe various proliferative changes, fibrocystic changes and stromal changes in fibroadenoma. Some of these changes may be of clinical significance. Limited data is available on statistical analysis of these common changes in literature.

The mean age was 28 years approximately, which was similar to various other studies. ${ }^{10,11}$ Maximum number of cases were in second decade. Incidence of fibroadenoma decreases with increase in age. However, we found that the complex features were observed in second, third and fourth decades were $2.4 \%, 12.63 \%$ and $20 \%$ respectively. This proved that complex features increased with increasing age, thereby having positive predilection for malignancy in future. ${ }^{12-14}$ Similar results were disclosed by other study. ${ }^{15}$

Fibroadenoma with history of 18 months or below showed $(8.04 \%)$ more complex fibroadenomas, while the fibroadenoma with more than 18 months showed only 5 (3.49\%) cases of complex fibroadenoma, which shows there is no relationship of duration with complexity of fibroadenoma in our study. A study by H. Takei et al. strongly relates to the fact that fibroadenomas naturally lapse with increasing age, becomes smaller with time and most likely adopts complex histopathological features. ${ }^{16}$

On detailed HPE, we categorized the 
histomorphological changes into proliferative epithelial changes, fibrocystic epithelial changes, stromal changes and changes in adjacent parenchyma of fibroadenoma.

Among proliferative epithelial changes, epithelial hyperplasia is divided into three categories i.e. mild, moderate and florid according to the criteria of Page et al and Holland et al. ${ }^{15}$ We found maximum number of cases exhibiting mild ductal hyperplasia followed by moderate ductal hyperplasia and florid ductal hyperplasia. Epithelial ductal hyperplasia in fibroadenoma is reported by several authors, predominantly mild and moderate forms similar to our study. ${ }^{17-19}$ Whereas in some other studies moderate hyperplasia was more in comparison to mild hyperplasia. It is interesting in implying these relative risks to hyperplasia found within fibroadenomas, but nothing has been proven regarding this matter. ${ }^{20-22}$ However, if hyperplasia within fibroadenoma behaves in the same way as in the otherwise normal breast, it could make a contribution to the increased relative risk of malignancy associated with fibroadenoma and may be a reason for excision. No correlation with hyperplasia in the surrounding tissue was found. Therefore, the meaning of hyperplasia within fibroadenomas in terms of progression risks remains to be determined. ${ }^{23}$

The other proliferative changes comprised mainly of florid ductal hyperplasia, atypical hyperplasia, atypical lobular hyperplasia, Lobular carcinoma in situ (LCIS) and Ductal carcinoma in situ (DCIS). CIS arising within fibroadenoma was detected in $1.7 \% .25$ Few heterogeneous figures describing the occurrence of in situ and invasive carcinoma within fibroadenoma exist. Ozello and Gump found an incidence of $0.3 \%$ for invasive and in situ carcinoma taken together. ${ }^{14}$ Deschenes et al found one carcinoma in situ and one invasive carcinoma in 70 fibroadenomas. ${ }^{4}$ Further, Buzanowski et al reported five cases of LCIS in 4000 fibroadenomas $(0.1 \%) .{ }^{13} \mathrm{We}$ couldn't find any such case in our study. Early detection and removal of the fibroadenoma may be the reason for decrease in progression to atypical hyperplasia, CIS and invasive carcinoma.

Among fibrocystic epithelial changes, $35 \%$ of cases showed apocrine metaplasia similar to many other studies. This may be contributed to the large amount of slides available per case, as Azzopardi already stated; " no doubt more extensive sampling would reveal its presence even more frequently". ${ }^{24}$ Cystic changes were also seen in some of the cases followed by sclerosing adenosis, calcifications, microglandular adenosis, papilloma and pseudo-lactational changes. Our findings were similar to a study done by Kuijiper et al. ${ }^{25}$ Association of SA with cystic changes, apocrine metaplasia, fibroadenoma, intraductal papilloma as well with carcinoma breast is established in previous studies. ${ }^{25}$ In the current study we found total $13.6 \%$ $(n=43)$ cases of sclerosing adenosis, out of which $11 \%$ were related with cystic changes. All of them were fulfilling the criteria's of complex fibroadenoma.

Stromal changes revealed hyaline and myxoid changes in majority of cases. These changes are the most common to be encountered on HPE, but they do not have any further risk for malignant transformation. Intracanalicular pattern, pericanalicular pattern and mixed pattern was found, these are the basic histological patterns and are not associated with further risk of malignancy. Pseudoangiomatous like changes were seen in $0.63 \%(n=2)$. These changes are often misdiagnosed as pseudoangiomatous stromal hyperplasia (PASH) as observed by Kuijiper et al..$^{25}$ There was no evidence of multinucleated giant-cells, smooth muscle changes, infarct and mitotic figures in the current study.

We received only 71 cases of fibroadenoma along with adjacent parenchyma. As Kuijiper et al already stated, at least $0.5 \mathrm{~cm}^{2}$ of tissue should be present around the fibroadenoma to understand the various changes in the adjacent breast parenchyma. ${ }^{25}$ Out of 71 cases we got only 50 cases with adjacent parenchyma showed various changes like foci of tubular adenoma, inflammatory changes, granulation tissue and phyllodes tumor. Fat necrosis is usually seen due to post FNAC procedure whereas post-surgical resection leads to the formation of granulation tissue in the adjacent parenchyma of the breast. Two cases of fibroadenoma along with phyllodes tumor was comparable with a study by Nithya Thuruthiyath et al. ${ }^{26}$ Although it is not of much significance according various literatures. Kuijiper et al revealed LCIS and DCIS in few cases in their study but we could not found any case, which may be due to less availability of adjacent parenchyma as well due to less number of cases. ${ }^{25,8}$

\section{Conclusion}

In conclusion, fibroadenomas harboring complex features are known for malignant transformation. Extensive inventory of histopathological changes in epithelial and stromal elements are essential to be reported in the description of the report so as to alert the clinician for close follow up of the patient. This will help in appropriate timely management to reduce morbidity and mortality.

\section{Acknowledgement: Nil.}

\section{Conflicts of Interest: Nil}

\section{References}

1. Raouf A, Sun Y, Chatterjee S, et al., The biology of human breast epithelial progenitors. Semin Cell Dev Biol. 2012;23:606-9.

2. Ellis IO: Intraductal proliferative lesions of the breast: morphology, associated risk and molecular biology. Mod Pathol. 2010;23(2):1-7. 
3. Hutchinson WB, Thomas DB. et al. Risk of breast cancer in women with benign breast lesion. J Natl Cancer Inst. 1980;65:13-20.

4. Deschenes L, Jacob S, Fabia J, et al. Beware of breast fibroadenomas in middle-aged women. Can J Surg. 1985;28:372-7.

5. Pick PW, Iossifide IA. Occurrence of breast carcinomas within a fibroadenoma: a review. Arch Pathol Lab Med 1984;108:590-3.

6. Dupont WD, Page DL, Parl FF, et al. Long-term risk of breast cancer in women with fibroadenoma. $N$ Engl J Med. 1994;331-3.

7. Carter B.A., Page D.L., Schuyler P., Parl F.F., Simpson J.F., Jensen R.A. No elevation in long-term breast carcinoma risk for women with fibroadenomas that contain atypical hyperplasia. Cancer. 2001;92:30-36.

8. Kuijper, A et al. "Multiple Fibroadenomas Harbouring Carcinoma in Situ in a Woman with a Family History of Breast/ovarian Cancer." Journal of Clinical Pathology. 2002;55(10):795-797.

9. Rosen, PP. Rosen's Breast Pathology (3rd ed.) Philadelphia, Lippincott Williams \& Wilkins,2009;200-1.

10. Thakur Brijesh, Misra Vatsala et al.

"Clinicohistopathological features of Fibroadenoma Breast in patients less than 20 years of age and its comparison with elder patients." IJSR. 2014;3(5):67-71.

11. Ullah N, Israr M, Ali M. Evaluation of Benign Breast Lump. Pak J Surg 2010;26:261-4.

12. Diaz NM, Palmer JO, McDivitt RW. Carcinoma arising within fibroadenomas of the breast, A clinicopathologic study of 105 patients. Am J Clin Pathol. 1991;95(5):61422.

13. Fondo EY, Rosen PP, Fracchia AA, Urban JA. The problem of carcinoma developing in a fibroadenoma: recent experience at Memorial Hospital. Cancer. 1979;43(2):563-567.

14. Pick PW, Iossifides IA. Occurrence of Breast Carcinoma within a fibroadenoma. A review. Arch Pathol Lab Med. 1984;108:590-596.

15. Page DL, Anderson TJ, Rogers LW. Epithelial hyperplasia and carcinoma in situ (CIS). In: Page DL, Anderson TJ, eds. Diagnostic Histopathology of the Breast. Edinburgh, Scotland: Churchill Livingstone; 1987;120-92.
16. H Takei, Y Iino, J Horiguchi et al. Natural History of Fibroadenomas Based on the Correlation Between Size and Patient Age. Jpn J Clin Oncol. 1999; 29 (1):8-10.

17. $\mathrm{Wu}, \mathrm{Yu}$-Ting et al. "Breast Cancer Arising within Fibroadenoma: Collective Analysis of Case Reports in the Literature and Hints on Treatment Policy." World Journal of Surgical Oncology. 2014;12:335-40.

18. Moskowitz M, Gartside P, Wirman JA, et al. Proliferative disorders of the breast as risk factors for breast cancer in a self- selected screened population: pathologic markers. Radiology. 1980;134:289-291.

19. Goodman ZD, Taxy JB. Fibroadenomas of the breast with prominent smooth muscle. Am J Surg Pathol 1981;5:99101.

20. Spagnolo DV, Shilkin KB. Breast neoplasms containing bone and cartilage. VirchowsArch. 1983;400:287-95.

21. O'Hara MF, Page DL. Adenomas of the breast and ectopic breast under lactational influences. Hum Pathol. $1985 ; 16: 707-12$

22. Rosen PP. Fibroepithelial neoplasms. In: Rosen PP, ed. Breast pathology. Philadelphia, PA: Lippincott-Raven, 1997;143-75.

23. Noguchi S, Yokouchi H, Aihara T, et al. Progression of fibroadenoma to phyllodes tumor demonstrated by clonal analysis. Cancer 1995;76:1779-85.

24. Azzopardi JG, Ahmed, Mills RR. In: Major problems in pathology. Benington JC, editor. Edinburgh: WB Saunders Company Ltd; Problems in Breast Pathology; 1979;325-8.

25. Kuijper A, Mommers EC, van der Wall E, et al. Histopathology of fibroadenoma of the breast. Am J Clin Pathol 2001;115:736-742.

26. Thuruthiyath, Nithya et al. "Giant Fibroadenoma of Breast in an Adolescent Girl." Oman Medical Journal. 2012;27(4):314-5.

How to cite this article: Ansari JN, Buch AC, Pandey A, Ruby R, Siddique A. Spectrum of histopathological changes in fibroadenoma of breast. Ind J Pathol Oncol, 2018;5(3):429-434. 